This is an electronic reprint of the original article. This reprint may differ from the original in pagination and typographic detail.

Author(s): Hyrkäs, Markku; Apaja, Vesa; Manninen, Matti

Title: Many-particle dynamics of bosons and fermions in quasi-one-dimensional flat-band lattices

Year: $\quad 2013$

Version:

Please cite the original version:

Hyrkäs, M., Apaja, V., \& Manninen, M. (2013). Many-particle dynamics of bosons and fermions in quasi-one-dimensional flat-band lattices. Physical Review A, 87(2), Article 023614. https://doi.org/10.1103/PhysRevA.87.023614

All material supplied via JYX is protected by copyright and other intellectual property rights, and duplication or sale of all or part of any of the repository collections is not permitted, except that material may be duplicated by you for your research use or educational purposes in electronic or print form. You must obtain permission for any other use. Electronic or print copies may not be offered, whether for sale or otherwise to anyone who is not an authorised user. 


\title{
Many-particle dynamics of bosons and fermions in quasi-one-dimensional flat-band lattices
}

\author{
M. Hyrkäs, ${ }^{1}$ V. Apaja, ${ }^{1}$ and M. Manninen ${ }^{2}$ \\ ${ }^{1}$ Nanoscience Center, Department of Physics, FIN-40014 University of Jyväskylä, Finland \\ ${ }^{2}$ Rector's Office, FIN-40014 University of Jyväskylä, Finland
}

(Received 21 March 2012; revised manuscript received 20 December 2012; published 15 February 2013)

\begin{abstract}
The difference between boson and fermion dynamics in quasi-one-dimensional lattices is studied by calculating the persistent current in small quantum rings and by exact simulations of the time evolution of the many-particle state in two cases: expansion of a localized cloud and collisions in a Newton's cradle. We consider three different lattices which in the tight-binding model exhibit flat bands. The physical realization is considered to be an optical lattice with bosonic or fermionic atoms. The atoms are assumed to interact with a repulsive short-range interaction. The different statistics of bosons and fermions lead to different dynamics. Spinless fermions are easily trapped in the flat-band states due to the Pauli exclusion principle, which prevents them from interacting, while bosons are able to push each other out from the flat-band states.
\end{abstract}

DOI: 10.1103/PhysRevA.87.023614 PACS number(s): 03.75.Lm, 05.30.Fk, 05.30.Jp, 71.10.Fd

\section{INTRODUCTION}

Crystal structures existing in nature exhibit fascinating band structures which determine electronic, optical, magnetic, and thermal properties of materials. The curvature of an energy band determines the effective mass of the electron, which can be hundreds of times the normal mass, such as in heavy fermion materials [1], or infinite, such as in the kagomé lattice [2] and other flat-band lattices [3,4], or even zero, such as in graphene [5].

Quantum-dot lattices for electrons [6-8] and optical lattices for atoms [9-12] have provided a new experimental setup where the lattice structure can be formed artificially and, consequently, structures which do not exist in nature can also be experimentally studied and utilized. Moreover, in optical lattices, atom dynamics can be studied without problems caused by lattice defects or phonons $[9,13,14]$ and the atoms trapped in the lattice can be chosen to be fermions or bosons.

The manipulation of the parameters of the optical lattice and the properties of the atoms allows variation of the magnitude and even the sign of the hopping parameters between neighboring lattice sites [15].

In this paper, we will study quasi-one-dimensional (Q1D) lattices with flat bands. Our motivation is the fast development in the research of atoms trapped in optical lattices, which has shown that surprisingly complicated lattice structures can be manufactured, such as the kagomé lattice [16]. Recently, we suggested how a flat band exhibiting a $2 \mathrm{D}$ edge-centered square lattice could be made [17]. Such systems can be quite accurately described with a Hubbard Hamiltonian with contact interaction between the atoms. In the limit of an infinitely strong interaction, only one atom can occupy each lattice site and, consequently, bosonic atoms will also have an "exclusion principle" in the simple (localized) tight-binding basis of single-particle states. However, bosons and fermions are different due to the different symmetry of the manyparticle wave function. In this case of spinless particles, the fermion wave function is a single Slater determinant, while the boson wave function is much more complicated, consisting of many permanents. This causes interesting differences in the quantum dynamics of these two systems. For example, the time evolution of out-of-equilibrium bosons in 1D can be nonergodic [18]. The situation here is very different from that of a rotating two-dimensional harmonic trap where the vortex formation mechanisms in boson and fermion systems are closely related [19]. Trapped bosonic gas can be brought all the way to the infinite repulsion limit (Tonks-Girardeau gas), where bosons behave locally like free fermions-an effect known as fermionization-but their momentum distribution is not fermionic [20]. Flat bands add a very intriguing flavor to the dynamics, as atoms occupying such bands are essentially immobile, and allow us to contrast bosons and fermions. In a magnetic field, such immobility means localization in a so-called Aharonov-Bohm cage [21,22].

In Sec. II, we introduce three different Q1D lattices with flat bands. The simplest is a triangle lattice, which has a flat band that is separated by an energy gap from a normal band. In the diamond lattice, the flat band either cuts through two normal bands or can be separated from them. In the stub lattice, the flat band is between two normal bands that are separated from it.

In Sec. III, we introduce the many-body problem and describe how the dynamical simulations were made and the persistent currents calculated. In Sec. IV, we describe the results for the persistent current calculations, and in Sec. V, we present the results for the dynamical simulations. The conclusions are given in Sec. VI.

\section{QUASI-ONE-DIMENSIONAL FLAT-BAND LATTICES}

There exists a large number of different lattice structures which in the simple tight-binding model, with only one state per lattice site and only the nearest-neighbor hopping, exhibit band structures with one or more flat bands $[3,4,17]$. Often the reason for the flat band is a solution where the singleparticle wave function is zero at some connecting sites of the lattice, making it impossible for the particles to move through the lattice. This kind of lattice can be one, two, or three dimensional.

Figure 1 shows the Q1D lattices and the corresponding band structures studied in this paper. The single-particle 


\section{a) a $90-00-0$}
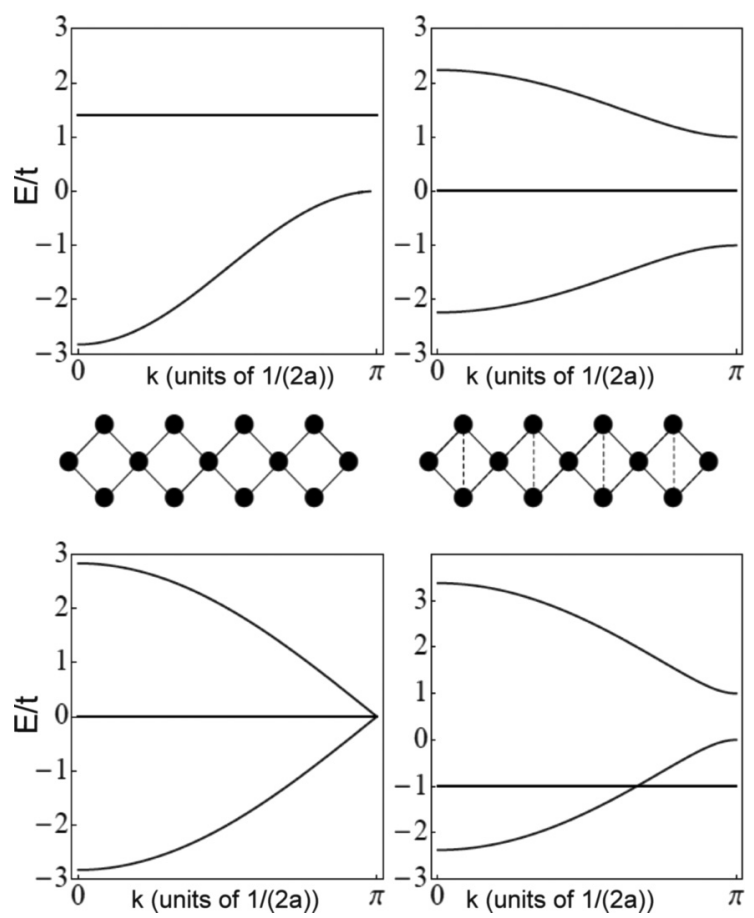

FIG. 1. Band structures of the Q1D lattices studied: triangle lattice, stub lattice, diamond lattice, and diamond lattice with transverse hopping. The vertical axis shows the energy in units of $t=1$ and the horizontal axis shows the $k$ value in units of $1 /(2 a)$, with $a$ being the lattice constant. In the triangular lattice, the hopping parameter shown as a dashed line has the value $t^{\prime}=1 / \sqrt{2}$. The parameter for the transverse hopping (dashed line) in the diamond lattice is $t^{\prime}=-1$.

Hamiltonian is the Hückel-type tight-binding (TB) model,

$$
\hat{H}_{\mathrm{TB}}=-\sum_{i, j}^{\mathrm{nn}} t_{i j} \hat{a}_{i}^{\dagger} \hat{a}_{j},
$$

where $\hat{a}_{i}^{\dagger}\left(\hat{a}_{i}\right)$ creates (destroys) a particle in lattice site $i, t$ is the hopping parameter, and "nn" means that the sum is taken only over nearest-neighbor sites. Throughout this paper, we use the natural units of the Hückel (or Hubbard) model where $t=1, m=1$, and $\hbar=1$.

In general, the hopping parameter $t_{i j}$ can vary in its magnitude and sign from site to site. This can also be realized in experiments, as was recently shown by Struck et al. [15]. This independent tuning of the lattice parameters allows one to move the flat band up or down in energy in the diamond lattice, and to make the flat-band triangular lattice discussed in this work.

In the triangle lattice, the ratio of the two hopping parameters has to be $t / t^{\prime}=\sqrt{2}$ in order to make one of the bands flat. For a positive $t$, the flat band is above the normal band, while for a negative $t$, it is below. In the stub lattice, the hopping parameter is the same between all neighbors. This lattice has a flat band in between two normal bands and is separated from them by gaps. The diamond lattice also has
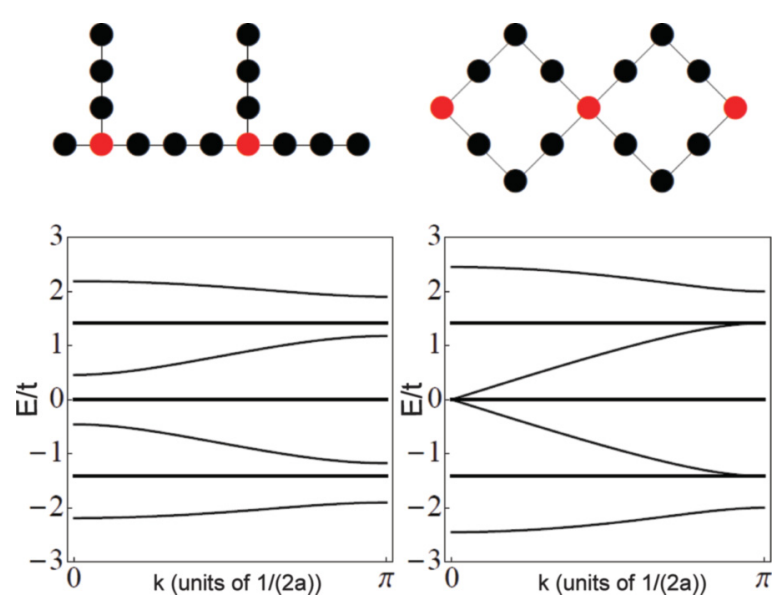

FIG. 2. (Color online) Band structures of extensions of the stub and the diamond lattices. The vertical axis shows the energy in units of $t=1$ and the horizontal axis shows the $k$ value in units of the inverse lattice constant. The gray nodal dots show the lattice sites at which the wave functions of the flat-band states are zero.

three bands with the flat band in the center, but in this case all the bands meet at the Brillouin-zone boundary. Adding a transverse hop between two points in each diamond, as shown as a dashed line in the bottom right panel of Fig. 1, moves the flat band in relation to the other two bands. At the same time, a gap opens between the two normal bands. For positive values of the transverse hopping parameter $t^{\prime}$, the flat band is raised, so that it either crosses the higher of the normal bands or is above both of them. Similarly, for negative values, the band is lowered (as show in the figure). Lowering the flat band can also be achieved by flipping the signs of all the hopping parameters, which inverts the band structure. This may be an easier configuration to realize in an experiment.

In the cases of the stub lattice and the diamond lattice, the flat band appears because the corresponding eigenstates have zero amplitude at the contact points of the unit cells, preventing any motion of particles from one unit cell to another. In the triangular lattice, the explanation is more subtle, as will be seen below when the persistent currents are considered.

The stub lattice and the diamond lattice can be generalized to Q1D lattices with several flat bands, as demonstrated in Fig. 2. In each flat band, the wave functions are zero at the corner points shown as gray dots. The positions of the flat bands are then determined by the length of the one-dimensional lattice (black dots) between the corner points. In both cases of Fig. 2, there are three sites between the corner sites. These sites can be thought to form a molecule with three sites and energy levels $(-\sqrt{2}, 0, \sqrt{2})$ in the tight-binding model. These energy levels determine the positions of the flat bands, which are then naturally the same in both lattices.

Next we consider how cold atoms in an optical lattice could be arranged to a diamond lattice structure. In experiments, one induces a dipole moment in the atoms with an oscillating electric light field from a laser. Interaction of the dipoles with standing electromagnetic waves creates the trapping potential for the optical lattice, as reviewed in Ref. [9]. Multiple lasers can be arranged to trap atoms in two- or three-dimensional optical lattices, where the trapping potential of each laser can 

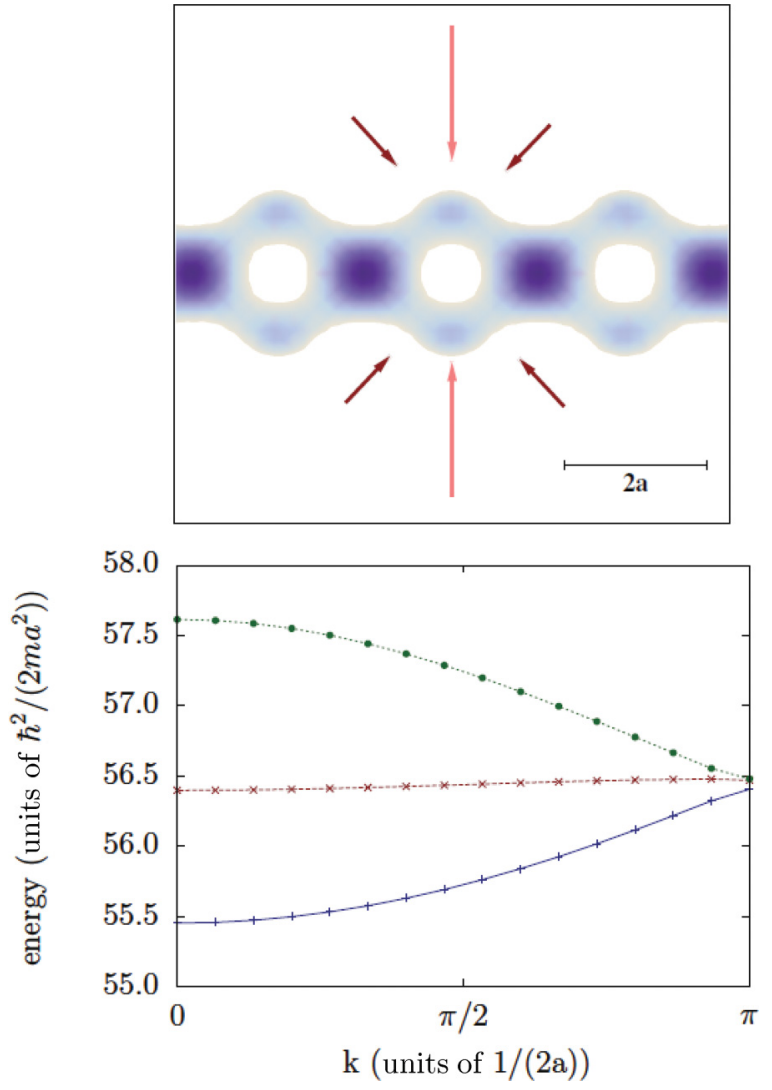

FIG. 3. (Color online) Upper figure: Suggested laser setup (with a harmonic confinement in the vertical, $y$ direction) to produce a diamond chain lattice, and the resulting optical potential given in Eq. (2). Darker areas denote lower potential. Lower figure: The three lowest tight-binding bands for the potential.

be described with a trigonometric function, $\cos ^{2}(x)$ or $\cos (2 x)$. One possible laser setup to create a linear diamond lattice is shown in Fig. 3, together with the optical potential. We solved the tight-binding band structure for the potential

$$
V(x, y)=V_{0}\left[\cos ^{2}(x+y)+\cos ^{2}(x-y)+1.042 \cos ^{2}(y)+y^{2}\right],
$$

where the last term is an extra harmonic confinement in the direction of the $y$ axis and $V_{0}=34.3 \hbar^{2} /\left(2 m a^{2}\right)$, where $2 a$ is the period along the diamond chain. The harmonic confinement is added to reduce the periodic structure in the $y$ direction to quasi one dimensional. The Laplacian in the Schrödinger equation was approximated with a five-point stencil. These constants make the second band very flat, with width of only $7 \%$ of the width of the lowest band $\left[0.073 \hbar^{2} /\left(2 m a^{2}\right)\right.$ vs $\left.0.95 \hbar^{2} /\left(2 m a^{2}\right)\right]$. Figure 3 shows the three lowest bands, obtained using 30 points in the $x$ direction and 60 points in the $y$ direction. With these choices, the band gap to the fourth band is already $13 \hbar^{2} /\left(2 m a^{2}\right)$.

\section{MANY-BODY DYNAMICS}

We consider atoms in an optical lattice and assume that the interaction between them has such a short range that it is only effective when the atoms are located in the same lattice site. We assume the atoms to be spinless or to be in the same spin state. In the case of fermionic atoms, this means that the system is spin polarized and thus each atom has the same $z$ component of spin. The many-body Hamiltonian describing the system is the Hubbard Hamiltonian

$$
\hat{H}=-\sum_{i, j}^{\mathrm{nn}} t_{i j} \hat{a}_{i}^{\dagger} \hat{a}_{j}+\sum_{i} V_{i} \hat{n}_{i}+\frac{U}{2} \sum_{i} \hat{n}_{i}\left(\hat{n}_{i}-1\right),
$$

where $\hat{n}_{i}=\hat{a}_{i}^{\dagger} \hat{a}_{i}, V_{i}$ is a local potential, and $U$ is the strength of the contact interaction.

In the case of the spinless fermions, the interaction term is irrelevant since the Pauli exclusion principle requires that each occupation $n_{i}$ is either zero or one. The many-body problem thus reduces to a single-particle problem. The many-body state is a Slater determinant made out of the single-particle wave functions, which are solutions of the tight-binding model.

In the case of bosons, the situation is more complicated. If the repulsive interaction is infinitely strong, we can assume that the occupation of each site cannot be more than one. Unfortunately, this does not completely remove the complexity of the many-body problem like in the case of fermions. However, we can still neglect the interaction term of the Hamiltonian by restricting the Fock space to those states which have an occupation of 0 or 1 in each lattice site.

In solving the many-particle Hamiltonian in the case of bosons, we use the localized basis in a finite length of the lattice and with a small number of particles. This restriction of the computation to small systems has the advantage of allowing an exact diagonalization of the Hamiltonian. Alternatively, we can use the basis of matrix product states (MPS), where increasing the matrix dimensions increases the overlap with the exact state. Large systems, where exact diagonalization is impractical, can still be approximated by MPS with reduced matrix dimensions. Both the ground-state representation as MPS and the time evolution of the state can be described using the time-evolving block decimation (TEBD) method by Vidal [23]. In TEBD, one chooses a target size of the matrices and, as time evolution increases entanglement and thus expands the matrices, one truncates the matrices back to target dimensions by throwing out the least important contributions. What is important is determined by means of repeated singular value or Schmidt decompositions. At least for a short time, the truncation error does not affect the results appreciably. TEBD propagates time step by step, leaving also a controllable time-step error in the results. In small systems, both of these methods produced the same results.

We are interested in the effect of the flat bands on the many-body dynamics. To this end, it is useful to know how the single-particle states are occupied in a given many-body state. In the case of fermions, this is trivial since the many-body state is a single determinant of the single-particle states. In the case of bosons, we can determine the occupations by changing the basis from the localized basis $(|\alpha\rangle)$, where the many-body solution is $|\Psi\rangle=\sum_{\alpha} A_{\alpha}|\alpha\rangle$, to the tight-binding (TB) basis $(\beta)$, where $|\Psi\rangle=\sum_{\beta} B_{\beta}|\beta\rangle$. ( $|\alpha\rangle$ and $|\beta\rangle$ are Slater determinants or permanents made of the single-particle states.) The occupations can be found without resolving the coefficients $B_{\beta}$ by writing the creation operator of the TB basis as $\hat{b}_{j}^{\dagger}=\sum C_{j i} \hat{a}_{i}^{\dagger}$, where the coefficients $C_{j i}$ are obtained from 
the TB solution. The occupation of a TB basis state $k$ is then

$n_{k}=\left\langle\Psi\left|\hat{b}_{k}^{\dagger} \hat{b}_{k}\right| \Psi\right\rangle=\sum_{\alpha, \alpha^{\prime}} \sum_{i, j} A_{\alpha}^{*} A_{\alpha^{\prime}} C_{k i} C_{k j}^{*}\left\langle\alpha\left|\hat{a}_{i}^{\dagger} \hat{a}_{j}\right| \alpha^{\prime}\right\rangle$

The time dependence of the many-body state after a sudden change of the Hamiltonian (in our case, the local potential) can be determined by solving the ground state of the many-body problem (for the initial potential) and all the many-body states for the final potential, and expanding the initial state as

$$
|\Psi\rangle=\sum_{p} D_{p}\left|\Psi_{p}\right\rangle
$$

where $\left|\Psi_{p}\right\rangle$ is the $p$ th time-independent energy eigenstate of the final Hamiltonian. The time dependence now follows from the time dependencies of the final states which are known:

$$
|\Psi(t)\rangle=\sum_{p} D_{p} e^{-i E_{p} t}\left|\Psi_{p}\right\rangle
$$

where $E_{p}$ is the energy eigenvalue of the state $p$. Note that in the case of fermions each final state is a Slater determinant. It then follows that the time dependence of the many-body state can be determined by following the time dependencies of the individual single-particle states.

In principle, an initial local potential is actually not required, since one does not need to know the initial Hamiltonian, but only the initial state. One may, for example, prepare particles in certain lattice sites by any means conceivable, and then suddenly release them to follow the time evolution given by the final Hamiltonian.

\section{PERSISTENT CURRENTS}

Persistent currents in quantum rings with a few fermions have been extensively studied; for a review, see Ref. [24]. In the case of bosons, the early work was related to the research of macroscopic systems of ${ }^{4} \mathrm{He}[25,26]$, while lately several studies of persistent currents in toroidal traps of bosonic atoms have been reported [27-32].

There are several ways to produce a toroidal trap for atom condensates [27,33-36]. In the case of finite quantum rings made of the Q1D lattices considered, we induce an effective magnetic flux through the ring in order to induce a current. Neutral atoms do not interact with the magnetic flux in the same way as electrons in metallic or semiconducting quantum rings. However, laser fields can generate a phase change which has the same effect as a magnetic flux [37-39]. An effective flux can be created using rotationally symmetric LaguerreGaussian laser modes.

In the Hubbard model, a flux piercing the ring will cause a phase shift to the hopping parameter $t_{i j}$, changing it to $e^{i \Phi_{i j}} t_{i j}$. In the case of the triangle lattice, we have to notice that the phase shift $\Phi_{i j}$ is twice as large for the hop along the long edge of the triangle than along the short edges, i.e., the total phase shift is independent of the path of the particle from one point to another. In the case of the stub lattice, the phase shift along the stub is zero.

The persistent current can be determined as the derivative of the total energy with respect to the flux or by computing the expectation value of the current operator between two points:

$$
J=\frac{\partial E}{\partial \Phi} \quad \text { or } \quad J=\langle\hat{J}\rangle=\left\langle i \sum_{i, j}^{\mathrm{nn}} t_{i j} e^{i \Phi_{i j}} \hat{a}_{i}^{\dagger} \hat{a}_{j}\right\rangle .
$$

In the case of bosons with infinitely strong contact interaction $(U \rightarrow \infty)$ and no on-site potentials $\left(V_{i}=0\right)$, the Hamiltonian is the same for particles and holes, i.e., $\hat{H}=\sum \hat{a}_{i}^{\dagger} \hat{a}_{j}=$ $\sum \hat{a}_{j} \hat{a}_{i}^{\dagger}$, since the operators commute when $i \neq j$. This means that the ground-state energy and the persistent current are symmetric with respect to particles and holes, irrespective of the symmetry of the single-particle spectrum. The situation is different for fermions due to the anticommutation rule, which changes the sign of the Hamiltonian for holes. Consequently, in the case of fermions, the many-body energy and the persistent current are symmetric with respect to particles and holes only if the single-particle spectrum is symmetric.

In the strictly one-dimensional case, both boson and fermion systems are exactly solvable via the Bethe ansatz [40-43]. However, already in the strictly 1D case, bosons and fermions differ due to the different symmetry of the wave function [44]. In both cases, the current is a periodic function of the flux through the ring, but depending on the number of particles, the periodicity can have a different phase for fermions and bosons. In the case of a zero flux, the lowest-energy state for any number of bosons has zero angular momentum, while for an odd number of spinless fermions, the lowest-energy state has a finite angular momentum $(L=N / 2)$ $[24,32]$, resulting in a finite current with an infinitesimal flux.

In the strictly one-dimensional case, spinless fermions cannot pass each other. The same is true for bosons interacting with an infinitely strong $\delta$-function interaction. The flat-band lattices are necessarily quasi one dimensional and thus more complicated.

We study the persistent currents in the flat-band lattices by exactly diagonalizing the Hubbard Hamiltonian for rings with a small number of lattice sites. For bosons, we assume an infinitely strong contact interaction, and for fermions, we assume the system to be spin polarized. Figure 4 shows the results for rings made of the stub and the diamond lattices. In each case, the ring has 12 sites and from 1 to 11 atoms (for 12 particles, all of the sites are occupied and no current can flow). We notice that the bosonic and the fermionic cases are markedly different. The boson current shows the particle-hole symmetry mentioned above, i.e., the result is the same for $N$ atoms and for $12-N$ atoms.

In the case of fermions, the current is independent of the particle number when the flat band is being filled. This is because the system is noninteracting and the flat band cannot conduct. The $N=2$ case has a finite current already at zero flux. This is because in the single-particle picture this state has an angular momentum of 1 (or -1 ) and thus a current. In the case of the stub lattice, the current for four particles and small flux is zero because the lowest band is full. In the case of the diamond lattice, both normal bands meet the flat band at the Brillouin-zone boundary. Due to this degeneracy, we get a finite current for an infinitely small flux for particle numbers $N=4, \ldots, 8$. 

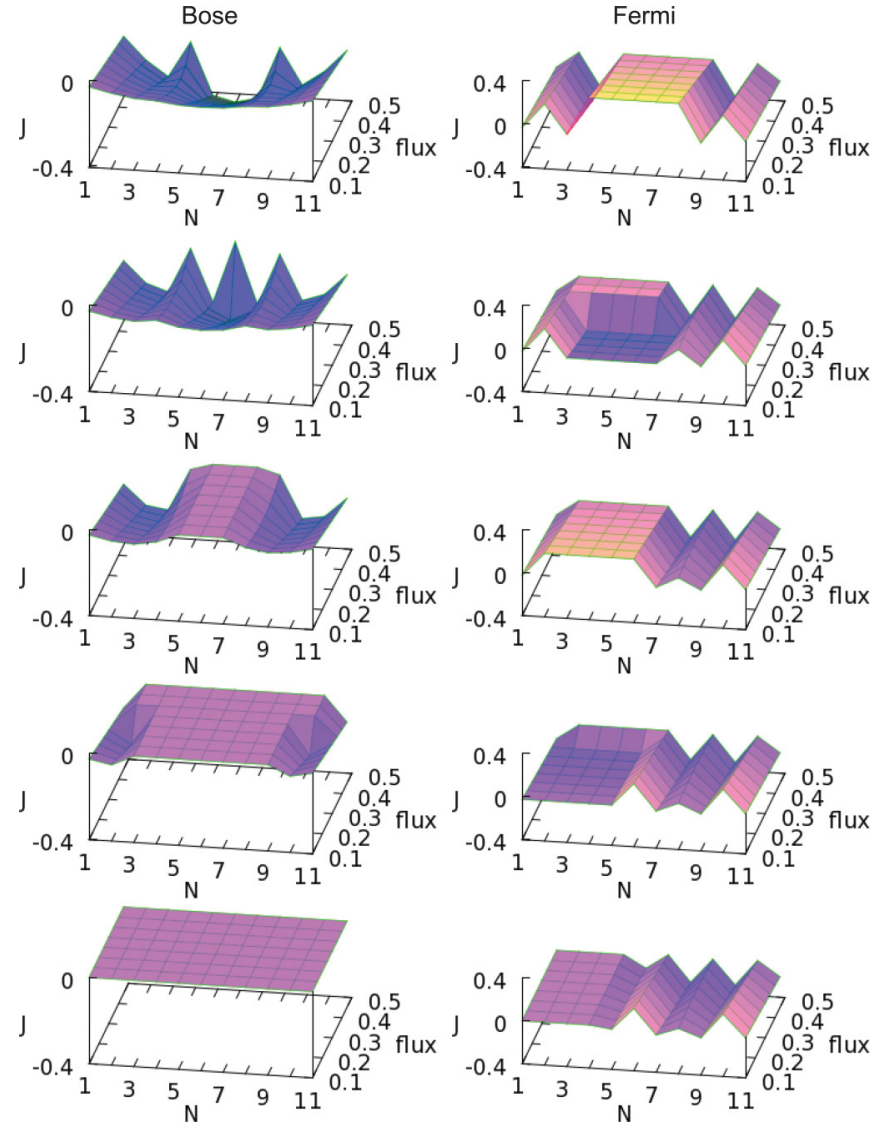

FIG. 4. (Color online) Persistent current of bosons (left) and fermions (right) in a quantum ring made of four unit cells of the diamond lattice. The current $J$ is shown as a function of the number of particles in the ring $(N)$ and the flux. In the upmost figures, the flat band is in between the normal bands, and, finally, in the lowest figures, it is below the normal bands (controlled by the value of the transverse hopping parameter, using values $t^{\prime}=0,-1.0,-1.5,-1.8,-2.1$ ). As the flat band moves down in energy, bosons occupy it more and more, which finally leaves all the bosons immobile. As particles are added, the fermion current's sign alternates, except during the filling of the flat band when it remains constant. The stub lattice gives results similar to the upmost figures.

\section{A. Diamond lattice}

The lowest panel in Fig. 4 shows the results for the diamond lattice where the transverse hopping shown in Fig. 1 has the value $t^{\prime}=-2.1$, which brings the flat band below both of the normal bands. In this case, the persistent current for bosons is always zero. For fermions, it is zero only for particle numbers $N=1, \ldots, 4$, which fit in the flat band. For particle numbers 6 and 10, the ground state is degenerate and the current starts from a finite value.

In the case of bosons, the situation where the flat band is low in energy is trivial, with most bosons occupying the flat band and becoming immobile. However, if the flat band is in the midst of the normal bands, the situation becomes more interesting. Looking at Fig. 4, one notices that the current is qualitatively different for some atom numbers. The reason can be traced back to rearrangement of single-particle energies and occupation.

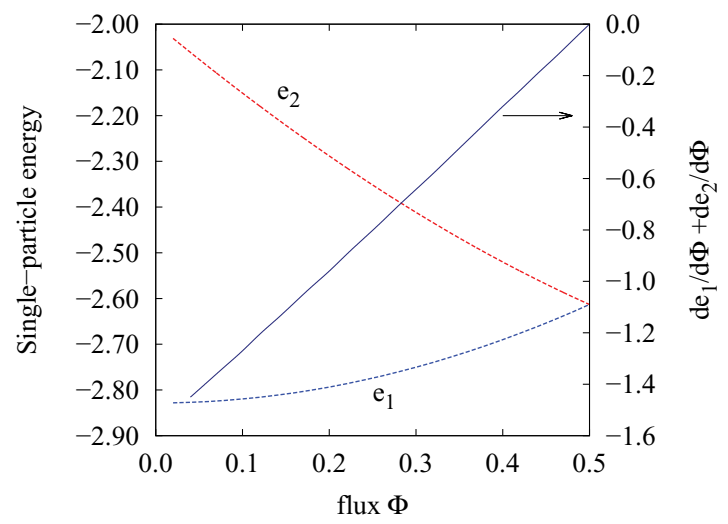

FIG. 5. (Color online) Energies of the two lowest single-particle states $e_{1}$ and $e_{2}$ (left $y$ axis) and the sum of the single-particle currents $d e_{1} / d \Phi+d e_{2} / d \Phi$ (right $y$ axis) as functions of flux. The figure shows that currents carried by the single-particle states 1 and 2 are in opposite directions and become equal in magnitude at flux $\Phi=0.5$.

First of all, the infinitely repulsive on-site potential does not show up while calculating the total energy of bosons with site occupations of 0 or 1 . Thus, their total energy is simply the sum of the single-particle energies, $E=\sum_{i} n_{i} e_{i}$, where $n_{i}$ are obtained from (4). As a consequence, the persistent current is the sum of "single-particle currents," using (7),

$$
J=\frac{d E}{d \Phi}=\sum_{i} n_{i} \frac{d e_{i}}{d \Phi} .
$$

Figure 5 shows that the single-particle currents $\frac{d e_{i}}{d \Phi}$ of states 1 and 2 circulate in opposite direction (positive and negative slope, respectively) and become equal in magnitude at flux $\Phi=0.5$, where the two states are also degenerate. This is true also for state pairs $(3,4),(9,10)$, and $(11,12)$. States $5-8$ are on a flat band and do not carry any current.

Next we want to see how the single-particle occupations change from $N=3$ to $N=5$. We choose a flux slightly below 0.5 in order to see the almost-degenerate case. The upper panel of Fig. 6 shows in detail the persistent current for flux $\Phi=$ 0.495 . For this flux, there is a very small current at $N=4$ and $N=8$. The lower panel of Fig. 6 shows that the nearly vanishing current for 4 atoms corresponds to almost equal occupations in the nearly-degenerate state pairs $(1,2),(3,4)$, $(9,10)$, and $(11,12)$, so the opposite contributions to the current cancel. Here the flat-band occupation is not directly relevant. Another way to see why $N=4$ is special is to note that there is exactly one atom per unit cell of three sites. The case $N=8$ has one hole per unit cell. Accordingly, for a diamond ring with $R$ sites, the boson persistent current vanishes at $N=R / 3$ and $N=R-R / 3$.

\section{B. Triangle lattice}

The results for the triangle lattice are shown in Fig. 7. We show results for cases where the flat band is above the normal bands (positive $t^{\prime}$ ) and where the flat band is below the normal bands (negative $t^{\prime}$ ). Note that the band structure does not depend on the sign of $t$.

In both cases, the current for bosons has a particle-hole symmetry, i.e., the current is the same for $N$ and $12-N$ 

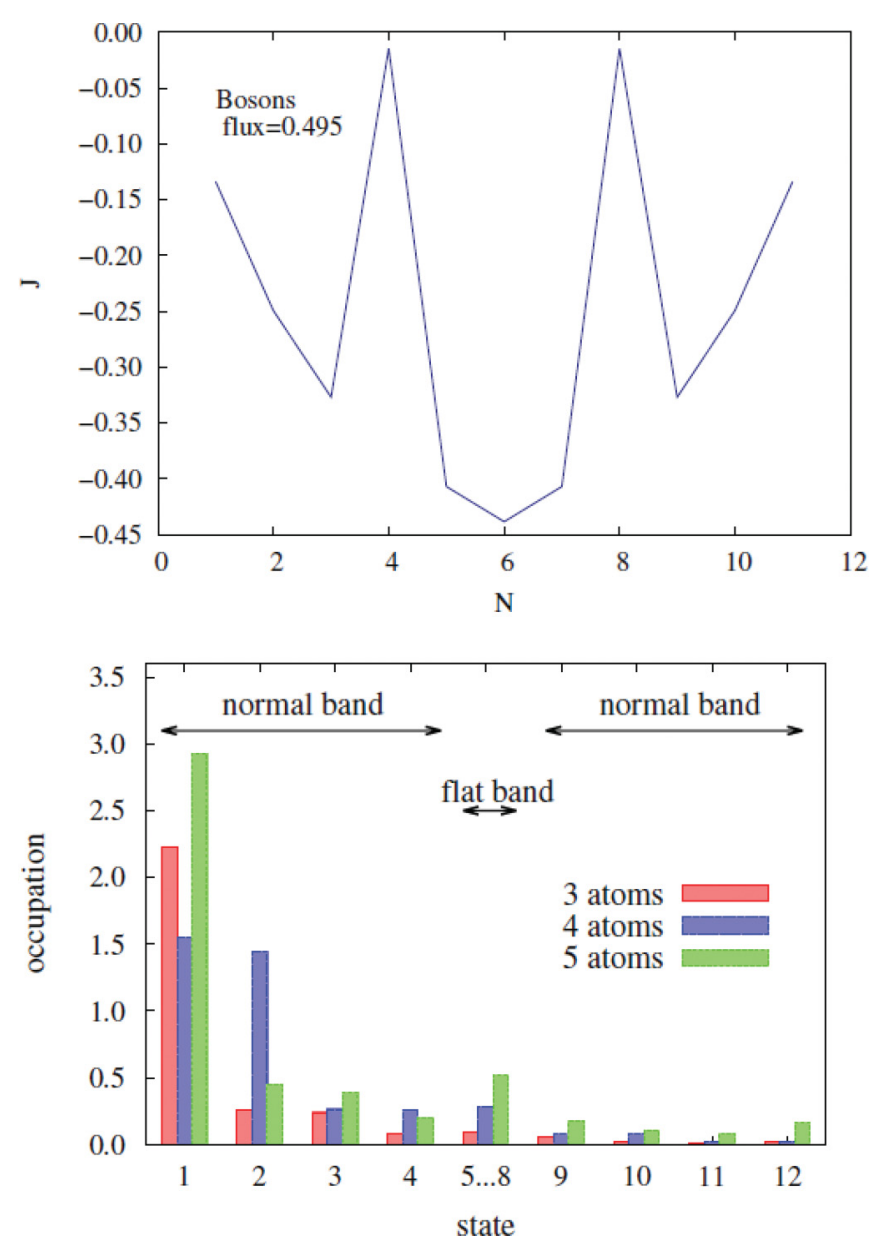

FIG. 6. (Color online) The upper figure shows the persistent current $J$ of bosons in a ring made of four unit cells of the diamond lattice as a function of the number of particles in the ring $(N)$ for flux $\Phi=0.495$. At $N=4$ and $N=8$, the current is qualitatively different. The lower figure shows how the occupation of the single-particle states evolves as $N$ increases from 3 to 5. Flat-band states 5-8 are degenerate. States 1 and 2 are almost degenerate, as are state pairs $(3,4),(9,10)$, and $(11,12)$.

particles, as discussed in the previous section. This symmetry causes a surprising effect when the flat band is at the bottom: The boson current is zero for all flux values, not only for small particle numbers $(N=1,2$, and 3$)$ but also for large particle numbers $(N=9,10$, and 11$)$.

The triangle lattice has the interesting feature that even when the total persistent current is zero, there is a current going around in each triangle. This is demonstrated in the case of two particles in Fig. 8, which shows separately the currents going along the short edge and the long edge of the triangle. When the flat band is at the bottom, the total current is zero, but the currents going along the short and long edges are nonzero with opposite signs. This means that the flux, which is zero inside the triangles, still induces a current going around each triangle.

In the case where the flat band is at the top, the currents along the short and long edges of the triangles go to the same direction, but have different magnitudes. Figure 8 also demonstrates that the currents are periodic functions of the flux. In the fermionic case, there is a discontinuity at integer
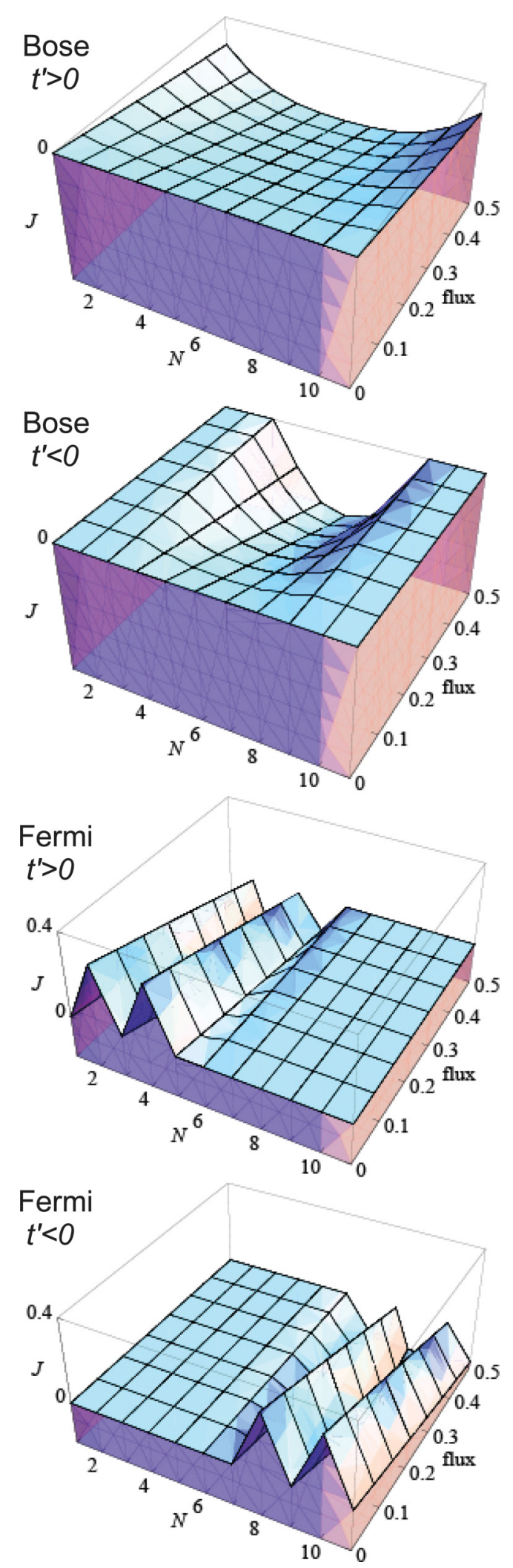

FIG. 7. (Color online) Persistent current of bosons (upper figures) and fermions (lower figures) in a quantum ring made of six unit cells of the triangle lattice. The current (vertical coordinate) is shown as a function of the number of particles in the ring $(N)$ and the flux. Note the different scale of the current for bosons and fermions. In the case $t^{\prime}>0$, the flat band is above the normal bands, while in the case $t^{\prime}<0$, it is below the normal bands. 

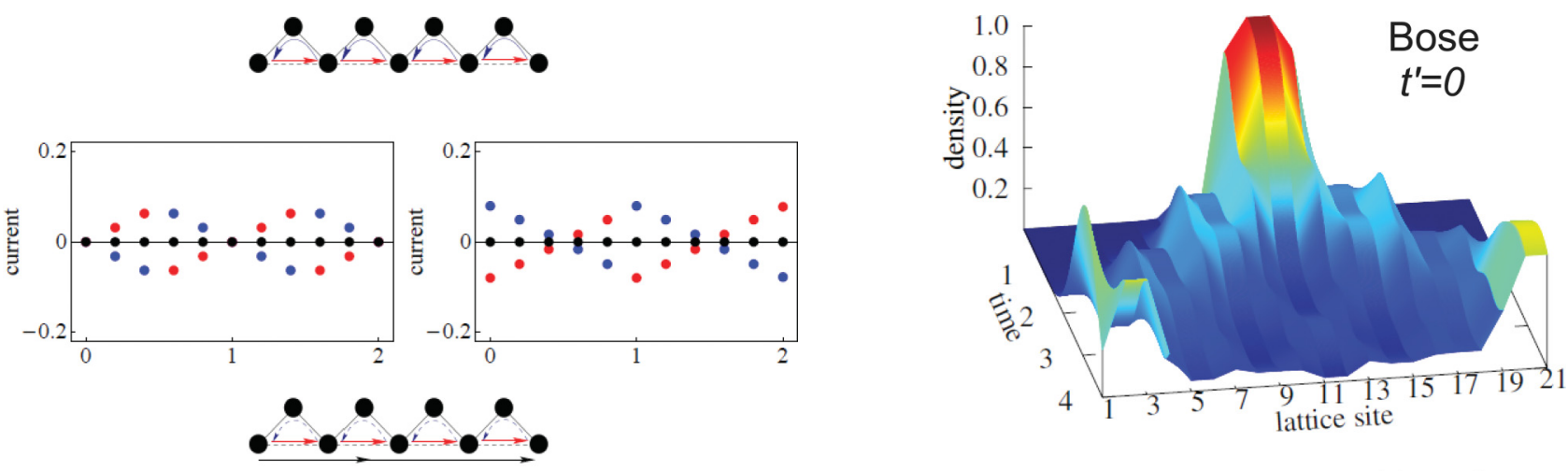
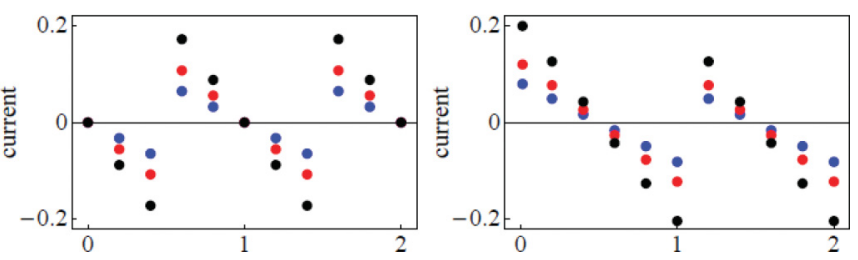

FIG. 8. (Color online) Current components of two fermions and bosons in a quantum ring of the triangular lattice. The black dots show the total current and the red and blue dots show the currents along the long edge and the short edge of the triangle, respectively. The left panels show the results for bosons and the right panels show the results for fermions. The upper panels are for the case where the flat band is below the normal band, resulting in zero net current. The lower panels are for the case where the flat band is above the normal band.

flux values, and in the bosonic case, there is discontinuity at half-integer flux values. These discontinuities are caused by the degeneracies of the many-body states.

\section{SIMULATIONS OF PARTICLE MOTION}

\section{A. Expansion of a localized cloud}

The results of the previous section show that for some particle numbers, the current through the ring is zero independently of the value of the flux. This suggests that the particles can be localized at the flat-band states. In order to study the localization further, we performed simulations of the dynamics. Initially, the particles were confined to a certain region of the lattice by adding a harmonic confinement $V_{\mathrm{h}}(i)=\alpha i^{2}$, where $i$ is the distance from the bottom of the harmonic confinement (in units of the distance between the lattice sites along the ring) and $\alpha$ is the strength of the potential. The unit of energy is $t$. Since we are interested only in qualitative differences, we chose $\alpha=1$.

We studied the dynamics of four particles in a diamond lattice with 21 sites and in a triangle lattice with 22 sites. In each case, we solved the lowest-energy state of the Hubbard Hamiltonian with the harmonic confinement, and fully diagonalized the Hamiltonian for the final state, i.e., without the harmonic potential. This allowed us [using Eq. (6)] to study how the particles move when the harmonic confinement is suddenly removed.

Figure 9 shows the results for the diamond lattice with 21 sites and periodic boundary conditions. The lattice sites were
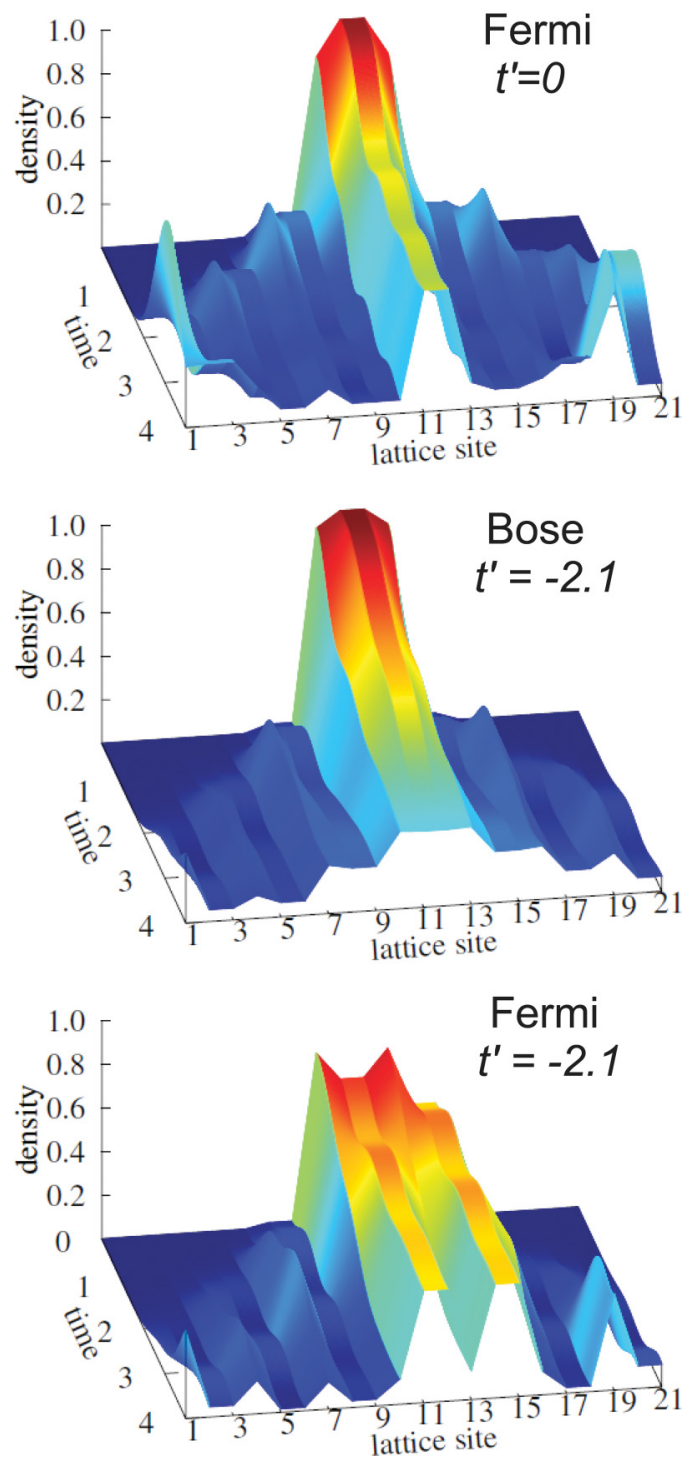

FIG. 9. (Color online) Particle density (occupation) at different lattice sites of the diamond lattice as a function of time after the harmonic confinement is removed. The figures show the results for bosons and fermions in two cases: In the case $t^{\prime}=0$, the flat band is in between the normal bands, and in the case $t^{\prime}=-2.1$, the flat band is below the normal bands.

numbered in succession so that the contact sites are $1,4,7$, 10 , etc. and the two other sites in each diamond are 2, 3, 5, 6, $8,9,11,12$, etc. The center of the harmonic confinement was set at the sites 11 and 12, so that the potential was 
$V_{11}=V_{12}=0, \quad V_{10}=V_{13}=1, \quad V_{9}=V_{8}=V_{14}=V_{15}=4$, and so on. Initially the particles are localized around the sites 11 and 12 using confinement $V(x)=\left(x-x_{0}\right)^{2}$, where $x_{0}$ is the linear coordinate of sites 11 and 12. It is convenient to use a linear coordinate along the quasi-two-dimensional chain so that sites at the tips of the diamond are at the same potential. When the potential is removed, the particles start to move outwards until they reach the borders of the simulation cell and start to overlap with the particles arriving from the neighboring cells (due to the periodic boundary conditions).

The two upper panels show the results for the case $t^{\prime}=0$ where the flat band is in between the normal bands. In this case, all of the bosons become mobile and fly away, while some of the fermions stay at the sites 11 and 12 . The situation is not much different when $t^{\prime}=-2.1$ and the flat band is below the normal bands. Also in this case the bosons fly away, only more slowly. The initial fermion distribution is wider, but again some of the fermions stay immobile. We also computed the dynamics for the stub lattice with 21 sites. The results were qualitatively similar to those of the diamond lattice with the flat band at the center.

Figure 10 shows the results for the triangle lattice. In this case, we have 21 lattice sites and the center of the harmonic confinement is at site 11 . The initial potentials are $V_{11}=0$, $V_{10}=V_{12}=1, V_{9}+V_{13}=4, V_{8}=V_{14}=9$, and so on. The two upper panels of Fig. 10 show the case where the flat band is at the top, and the two lower panels show the cases where the flat band is at the bottom. The results are rather similar to those for the diamond lattice. In both cases, the boson distribution widens with time and all of the bosons eventually fly away. Also, in both cases, a part of the fermions stays localized.

We have repeated the dynamics simulations for different particle numbers and numbers of lattice sites, using both periodic boundary conditions and a finite-length lattice. In all cases, the results are qualitatively similar to those shown in Figs. 9 and 10.

The general result that some of the fermions stay localized in the flat-band states is easy to understand. Spinless fermions with contact interaction are equivalent to noninteracting fermions. Those initially occupying a flat-band state will stay there when the confinement potential is removed. The same is not true for bosons, which can push each other out from the flat-band states.

In the cases where the flat band is the lowest band, the initial boson and fermion distributions are slightly different due to the different statistics. In order to get more insight into the initial many-body state, we determined the single-particle state occupancies for bosons and fermions using Eq. (4). The single-particle states are the tight-binding eigenstates without the confining potential. The results are shown in Fig. 11. The figure shows that the many-body state of the Bose system has nearly as large occupancy of the flat-band states as the Fermi system. In fermion systems, those particles are immobile, but in boson systems, they interact with the particles in the normal band and become mobile.

\section{B. Newton's cradle and Bloch oscillations}

As we saw, depending on the occupation of states, flat-band systems can have both inert and mobile electrons. In principle,
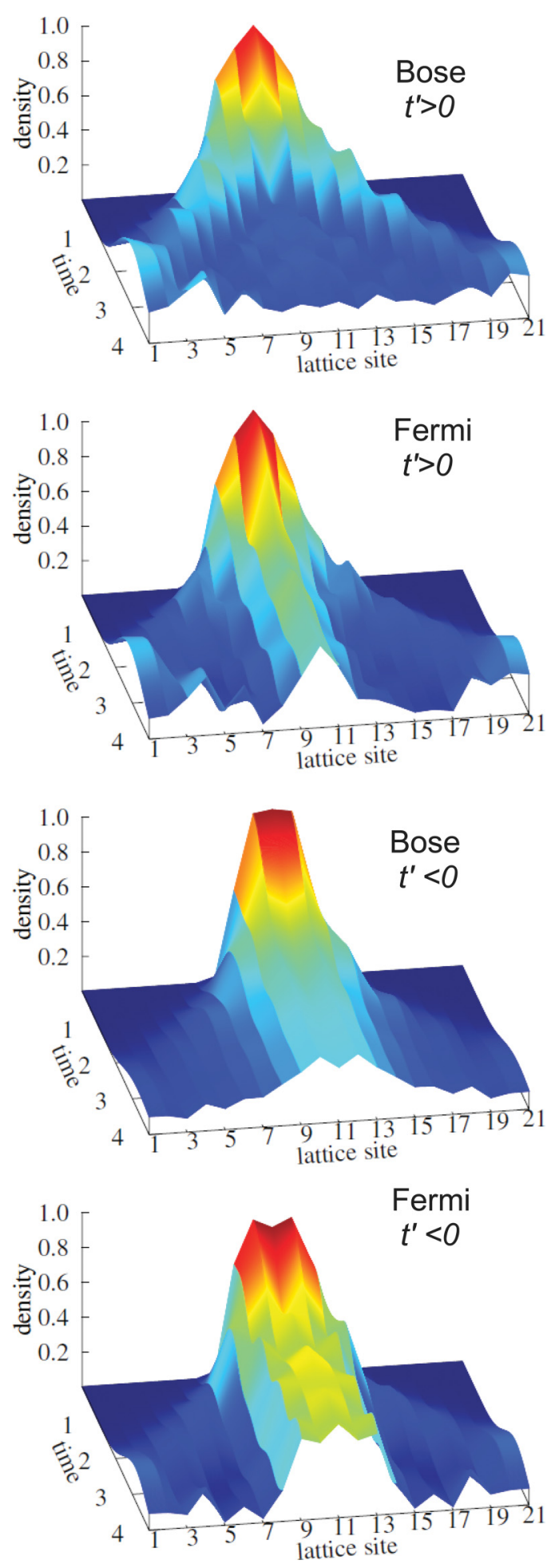

FIG. 10. (Color online) Particle density (occupation) at different lattice sites of the triangle lattice as a function of time after the harmonic confinement is removed. The figures show the results for bosons and fermions in two cases: In the case $t^{\prime}>0$, the flat band is above the normal bands, and in the case $t^{\prime}<0$, the flat band is below the normal bands.

this could resemble a quantum Newton's cradle, demonstrated in a trapped Bose gas by Kinoshita et al. [18]. Ideally, there is no dephasing, and perfect momentum exchange among indistinguishable atoms can leave some atoms immobile, just 

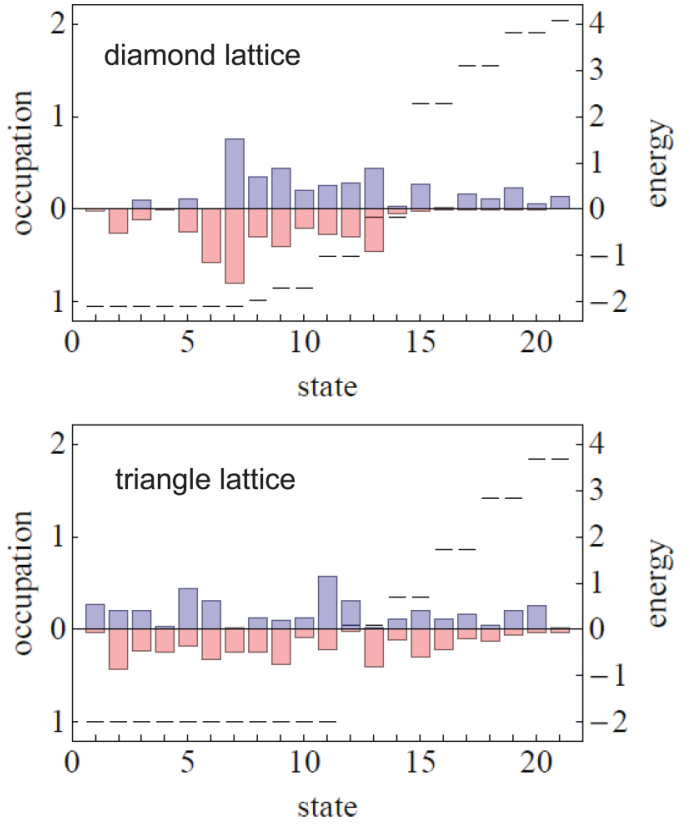

FIG. 11. (Color online) Occupation of single-particle states in the initial many-body state (in the presence of the harmonic confinement) for diamond and triangle lattices. The single-particle energy levels (without the confinement) are shown as short lines and the corresponding occupancies are shown as bars, above (blue) for bosons and below (red) the horizontal line for fermions. Here the flat band has the lowest energy.

like those on a flat band. A practical limitation to the realization of a Newton's cradle with atoms in a discrete lattice is posed by Bloch oscillations [45]: If one drives atoms with a too steep potential slope, they accelerate only to the point where their group velocity is so high that a Bragg reflection reverses their motion.

Let us look first at a basic linear chain. In Fig. 12, we have two atoms originally in the ground state of two harmonic potentials, with minima at sites 6 and 30. The plotted density scale is chosen to be from 0 to 0.4 , which covers the cases studied here. Subsequently, we remove the minimum at 6 and follow the time evolution of the density. The amplitude of the trapping potentials determines how localized the atoms are originally. The details of the initial states are not important, but after removing the minimum at 6 , the atom cloud around site 6 sits on a potential slope, the steepness of which is determined by the amplitude. With a shallow potential, we get a nice Newton's cradle where oscillations are only slightly distorted and damped. The damping is caused by many-body effects. If the potential slope is made too steep, however, Bloch oscillations set in before atom clouds have a chance to collide, and one can even have a rapidly oscillating cloud with no interference with the other cloud. In these examples, Bloch oscillations are symmetric, while in general they can be asymmetric [46].

Next we try something similar in a flat-band system. A suitably mutable flat-band system is the diamond chain with the vertical hopping parameter that shifts the flat-band level (see fourth panel in Fig. 1). Here the flat-band energy is chosen to be almost degenerate with the low edge of the lowest normal
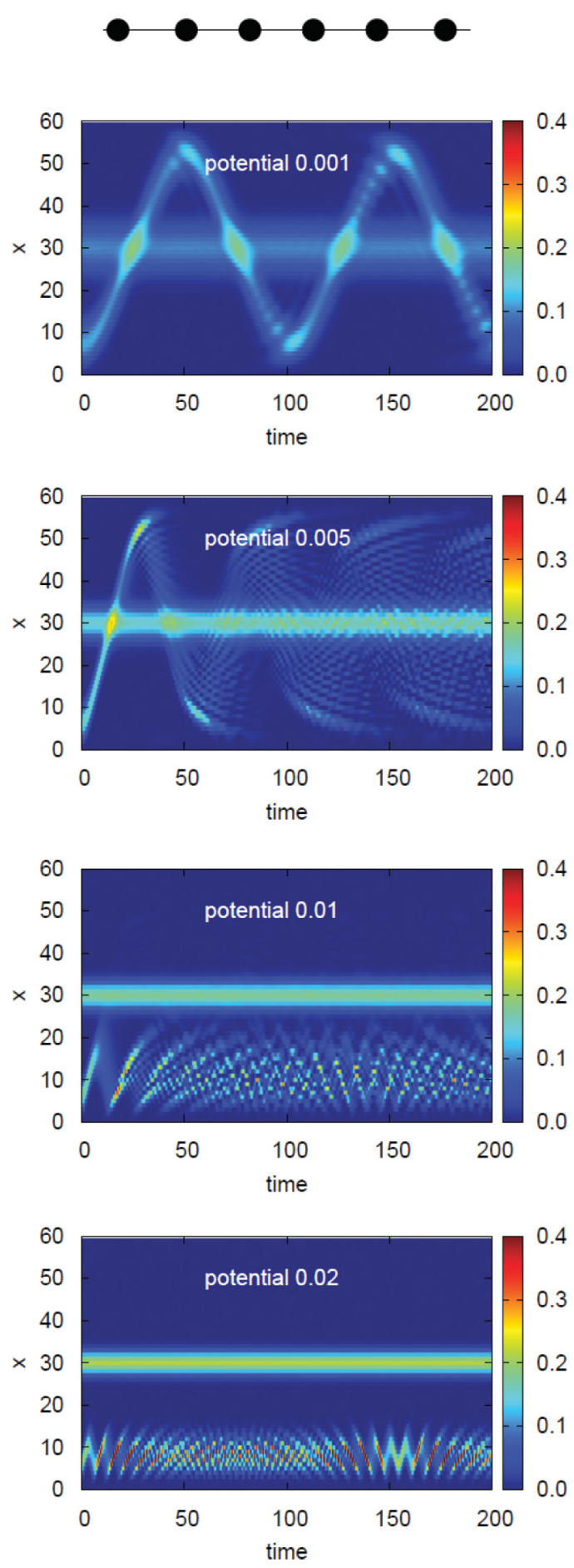

FIG. 12. (Color online) Attempts to make a Newton's cradle with two harmonic potentials easily end up creating two separate clouds due to Bloch oscillations. The figures represent a simple linear-chain density of two bosons originally in the ground state of a double harmonic well potential. They are set in motion by leaving only one minimum, with potential $V_{h}(i)=\alpha i^{2}$, where $i$ is the site index and $\alpha$ is the strength, as indicated in the figures. A shallow potential (upper left figure) sustains Newton's cradle oscillations for over 10 cycles. Time is measured in units of inverse energy $(\hbar=1)$.

band. We put the potential minima at $x=4$ and $x=20$, where $x$ is the coordinate along the diamond chain. In order to see the effect of the flat band, we want about $50 \%$ occupation on 

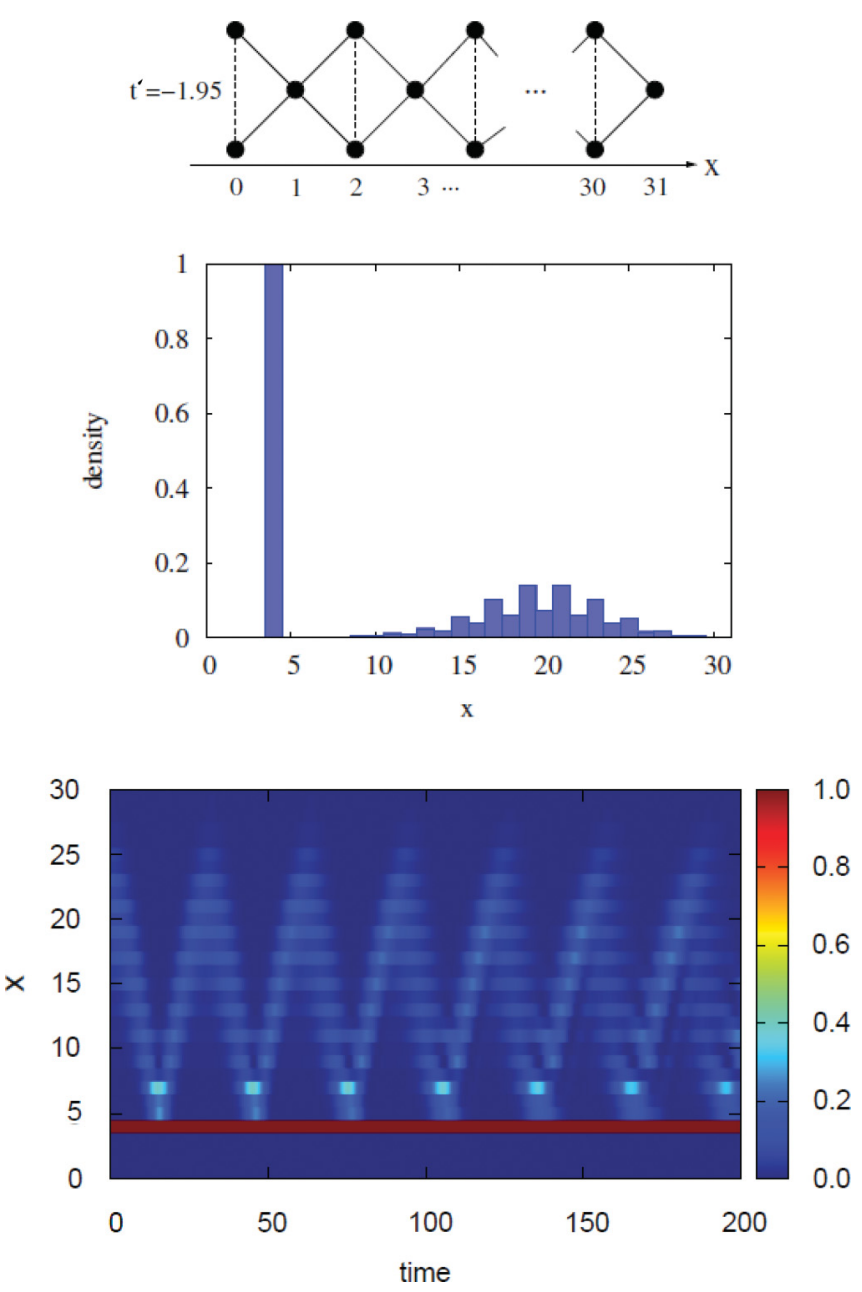

FIG. 13. (Color online) The upmost figure shows the diamond chain and the definition of the linear coordinate $x$. The middle figure shows the chosen initial density, with one atom on the left on the flat band, and the other one being less localized. After leaving only a shallow harmonic potential with minimum at $x=4$, the cloud on the right is driven left. The time evolution of the density is shown in the lowest figure, which shows how the occupied, low-energy flat band acts in this case as a reflective wall.

it. This is achieved by pinning one atom with a very strong potential to $x=4$, which in our chain has two sites (at the tips of the diamond). We want the other atom to remain mobile so we use a shallow potential with amplitude 0.001 to put it around $x=20$, but not on the flat band. Next we wish to set the latter atom in motion toward the first atom. The driving potential has to be very shallow to avoid Bloch oscillations. We use again a harmonic potential with amplitude 0.001 , which for the linear chain produced a nice Newton's cradle, with the minimum at $x=4$.

The result is presented in Fig 13-the plotted density ranges now from 0 to 1 because there is exactly one atom on the flat band. Instead of a Newton's cradle, we get a reflection from the flat-band atoms at $x=4$. These atoms are now held in place not by a potential, but by the flatness of the band. Such reflection is peculiar to flat-band systems, where a mere confinement as in the linear-chain case gives rise to momentum exchange in a Newton's cradle fashion. Also the unoccupied flat-band
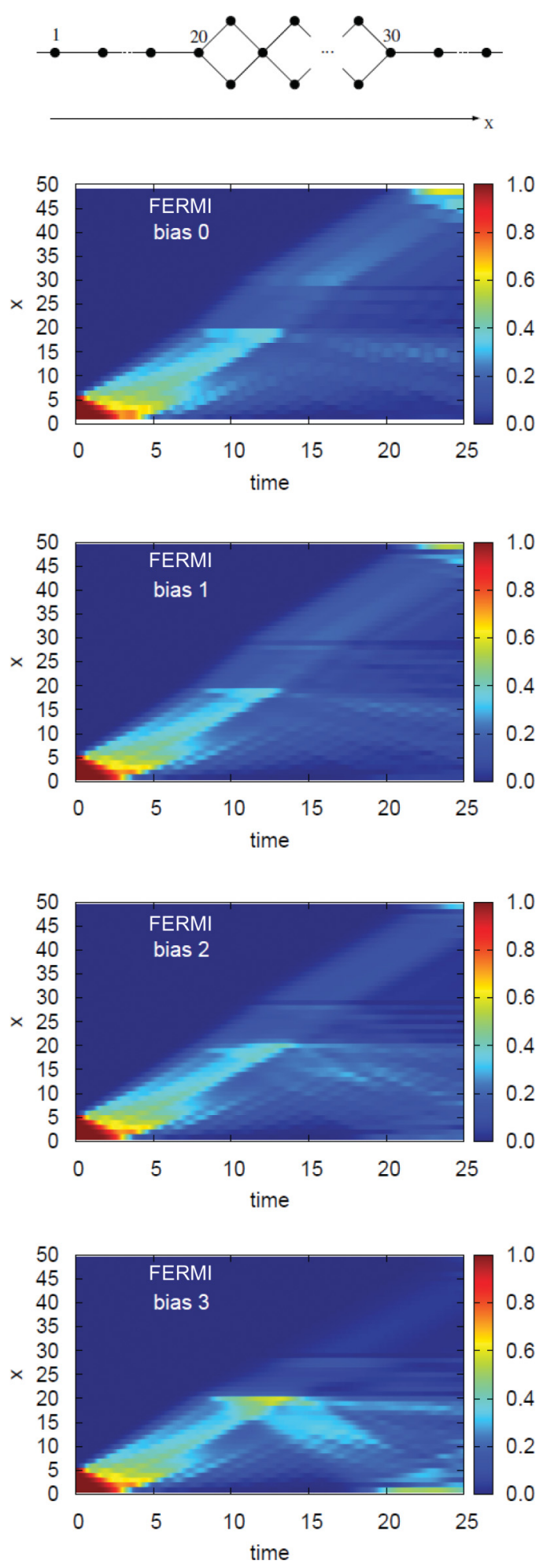

FIG. 14. (Color online) A diamond chain between two finite leads. Five fermions are initially on the leftmost sites and the potentials of the leads have been shifted by the bias value. The diamond chain is in the range $20<x<30$. The calculation was done using TEBD with $100 \times 100$ matrix product states.

states at low energy affect the motion of atoms: the horizontal stripes in the oscillating part are density maxima at the node points of the diamond lattice (odd- $x$ values). This is simply 

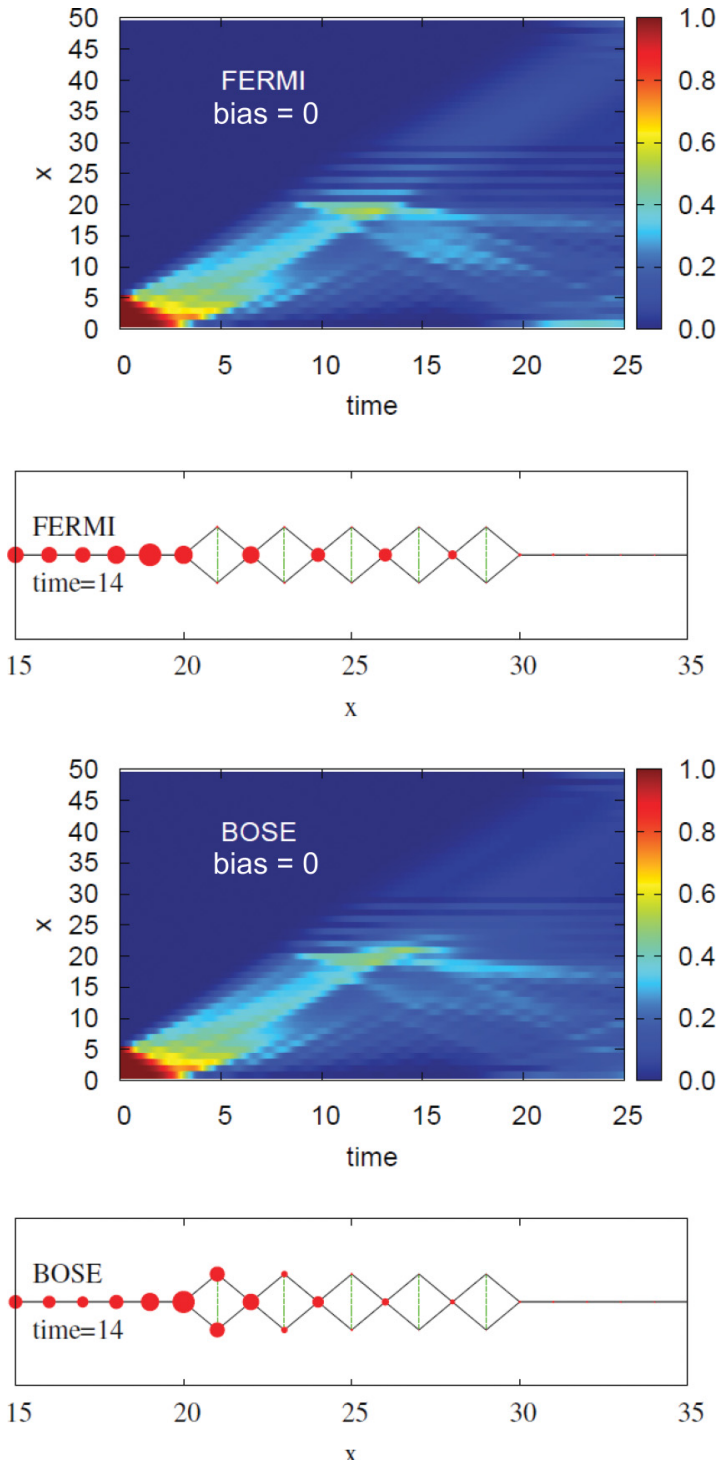

FIG. 15. (Color online) A diamond chain with transverse hopping between two finite leads. Apart from the modified band structure, the setup is the same as in Fig. 14. At time $=0$, the five bosons or fermions are at lead sites 1 to 5, and are set in motion by diffusion. The upper figures show the time evolution of the density for fermions and the lower figures show the time evolution of the density for bosons. Snapshots at time $=14$ show how sites are occupied by fermions and bosons; the circle size is proportional to the density at that site. Differences arise from the boson vs fermion statistics.

a consequence of the fact that while the atoms occupying a flat band now have no way of leaving the band and moving, there is also no way the moving atoms can occupy a flat band. As a byproduct, one could create a confining box in 1D by pinning two atoms to two well-separated even- $x$ sites (which puts them on a flat band). Other bosons in between would then be trapped by the two mirrors.

\section{Transport}

The transport properties of a $\delta$ chain between semi-infinite leads have been investigated in Ref. [47] by Schulze et al. in the case of adiabatic pumping, i.e., without external bias. In Fig. 14, we show an example of the time evolution in a flat-band system, now a diamond chain, between finite leads. Here the diamond lattice has normal bands symmetrically above and below the flat band (see Fig. 1). The potential difference between the left lead and the diamond lattice is given by the bias (e.g., for electrons, a voltage bias). For a small bias, fermions can occupy the low-energy normal band of the diamond chain and some reflect from the end of the the diamond chain. For a large bias, the potential step dominates and fermions reflect. In this setup, there is little difference between bosons and fermions.

The flat band can be shifted up or down in energy with respect to the normal bands by adding a transverse hopping $t^{\prime}$, as visualized in Fig. 1. Using the value $t^{\prime}=-2$, the flat band is pushed down to become the lowest band, degenerate with the lower edge of the lower dispersive band. Figure 15 shows again the time-dependent density. There is no bias; particle motion is now driven by diffusion.

To interpret Fig. 15, we note that the amplitude of the flat-band, single-particle state, which cannot be occupied by moving particles, is highest at the tips of the diamond (at $x=21,23, \ldots, 29)$. This throttles the motion of the particles, as they must use the normal band degenerate with the flat band. Fermions are affected more because of the Pauli principle (or the fermion phase factor, if one thinks of the current). Fermion density at the tips of the diamond remains always low, and they pile up in the nodal sites. This causes the horizontal stripes in the fermion density in Fig. 15, and is seen clearly in the snapshot at time 14 of the density at each site, which is shown in the same figure.

Up to approximately time 10, bosons diffuse to the diamond lattice almost exactly like fermions: they pile up in the nodal site at $x=22$ and tip sites remain almost empty. As mentioned above, the low-energy normal band is degenerate with the flat band. Boson occupation of these low-energy dispersive states is not limited, and bosons begin to fill the tip sites at $x=21$. This is clearly seen in the snapshot at time 14 in the lowest panel of Fig. 15. Due to the deflection to the tip sites, the diffusion of bosons through the diamond lattice is slowed down.

\section{CONCLUSIONS}

We have addressed the differences in particle dynamics between bosons and fermions in quasi-one-dimensional lattices with a flat band. The particles were assumed to be spinless and interacting with an infinitely strong contact interaction. In this case, the fermions are equivalent to noninteracting particles due to the Pauli exclusion principle. Consequently, the fermions occupying the flat-band states do not contribute to the persistent current in a quantum ring, and they are localized in the lattice.

In the case of bosons, the particles are truly interacting, making the system more interesting. The persistent current shows a particle-hole symmetry and the occupation of the flat band can also change the current. The bosons do not stay localized in the lattice, even if initially the partition of the many-body wave function to the single-particle states is essentially the same as in the case of fermions. 
These results are very general; they do not seem to depend on the detailed structure of the lattice or on the position of the flat band with respect to the normal bands with dispersion. This gains in importance only when the flat-band lattice is brought in contact with an external lattice.

\section{ACKNOWLEDGMENTS}

Ideas for this work were initiated by a workshop of the European Science Foundation research networking program POLATOM. We thank the Academy of Finland for financial support.
[1] G. R. Stewart, Rev. Mod. Phys. 56, 755 (1984).

[2] I. Syôzi, Prog. Theor. Phys. 6, 306 (1951).

[3] S. Deng, A. Simon, and J. Köhler, J. Solid State Chem. 176, 412 (2003).

[4] S. Miyahara, K. Kubo, H. Ono, Y. Shimomura, and N. Furukawa, J. Phys. Soc. Jpn. 74, 1918 (2005).

[5] A. H. Castro Neto, F. Guinea, N. M. R. Peres, K. S. Novoselov, and A. K. Geim, Rev. Mod. Phys. 81, 109 (2009).

[6] H. Lee, J. A. Johnson, M. Y. He, J. S. Speck, and P. M. Petroff, Appl. Phys. Lett. 78, 105 (2001).

[7] M. Koskinen, S. M. Reimann, and M. Manninen, Phys. Rev. Lett. 90, 066802 (2003).

[8] S. M. Reimann and M. Manninen, Rev. Mod. Phys. 74, 1283 (2002).

[9] I. Bloch, Nature Phys. 1, 23 (2005).

[10] A. J. Legget, Quantum Liquids: Bose Condensation and Cooper Pairing in Condensed Matter Physics (Oxford University Press, Oxford, UK, 2006).

[11] C. J. Pethick and H. Smith, Bose-Einstein Condensation in Dilute Gases, 2nd ed. (Cambridge University Press, Cambridge, UK, 2008).

[12] S. López-Aguayo, Y. V. Kartashov, V. A. Vysloukh, and L. Torner, Phys. Rev. Lett. 105, 013902 (2010).

[13] I. Bloch, Science 319, 1202 (2008).

[14] I. Bloch, J. Dalibard, and W. Zwerger, Rev. Mod. Phys. 80, 885 (2008).

[15] J. Struck, C. Ölschläger, R. Le Targat, P. Soltan-Panahi, A. Eckardt, M. Lewenstein, P. Windpassinger, and K. Sengstock, Science 333, 996 (2011).

[16] Gyu-Boong Jo, Jennie Guzman, Claire K. Thomas, Pavan Hosur, Ashvin Vishwanath, and Dan M. Stamper-Kurn, Phys. Rev. Lett. 108, 045305 (2012).

[17] V. Apaja, M. Hyrkäs, and M. Manninen, Phys. Rev. A 82, 041402 (2010).

[18] Toshiya Kinoshita, Trevor Wenger, and David S. Weiss, Nature (London) 440, 900 (2006).

[19] H. Saarikoski, S. M. Reimann, A. Harju, and M. Manninen, Rev. Mod. Phys. 82, 2785 (2010).

[20] Belen Paredes, Artur Widera, Valentin Murg, Olaf Mandel, Simon Folling, Ignacio Cirac, Gora V. Shlyapnikov, Theodor W. Hansch, and Immanuel Bloch, Nature (London) 429, 277 (2004).

[21] Julien Vidal, Benoit Douçot, Rémy Mosseri, and Patrick Butaud, Phys. Rev. Lett. 85, 3906 (2000).
[22] B. Douçot and J. Vidal, Phys. Rev. Lett. 88, 227005 (2002).

[23] G. Vidal, Phys. Rev. Lett. 93, 040502 (2004).

[24] S. Viefers, P. Koskinen, P. Singha Deo, and M. Manninen, Physica E 21, 1 (2004).

[25] J. D. Reppy and D. Depatie, Phys. Rev. Lett. 12, 187 (1964).

[26] W. Grobman and M. Luban, Phys. Rev. 147, 166 (1966).

[27] Y. Lyanda-Geller and P. M. Goldbart, Phys. Rev. A 61, 043609 (2000).

[28] T. Isoshima, M. Nakahara, T. Ohmi, and K. Machida, Phys. Rev. A 61, 063610 (2000).

[29] T. Wang, J. Javanainen, and S. F. Yelin, Phys. Rev. A 76, 011601 (2007).

[30] C. Ryu, M. F. Andersen, P. Cladé, V. Natarajan, K. Helmerson, and W. D. Phillips, Phys. Rev. Lett. 99, 260401 (2007).

[31] S. Bargi, F. Malet, G. M. Kavoulakis, and S. M. Reimann, Phys. Rev. A 82, 043631 (2010).

[32] V. E. Lembessis and M. Babiker, Phys. Rev. A 82, 051402 (2010).

[33] A. Hopkins, B. Lev, and H. Mabuchi, Phys. Rev. A 70, 053616 (2004).

[34] O. Morizot, Y. Colombe, V. Lorent, H. Perrin, and B. M. Garraway, Phys. Rev. A 74, 023617 (2006).

[35] P. F. Griffin, E. Riis, and A. S. Arnold, Phys. Rev. A 77, 051402 (2008).

[36] P. M. Baker, J. A. Stickney, M. B. Squires, J. A. Scoville, E. J. Carlson, W. R. Buchwald, and S. M. Miller, Phys. Rev. A 80, 063615 (2009).

[37] E. J. Mueller, Phys. Rev. A 70, 041603 (2004).

[38] L. Amico, A. Osterloh, and F. Cataliotti, Phys. Rev. Lett. 95, 063201 (2005).

[39] A. L. Fetter, Rev. Mod. Phys. 81, 647 (2009).

[40] E. H. Lieb and W. Liniger, Phys. Rev. 130, 1605 (1963).

[41] E. H. Lieb, Phys. Rev. 130, 1616 (1963).

[42] C. N. Yang, Phys. Rev. Lett. 19, 1312 (1967).

[43] E. H. Lieb and F. Y. Wu, Phys. Rev. Lett. 20, 1445 (1968).

[44] M. Manninen, S. M. Reimann, and S. Viefers, Physica E 46, 119 (2012).

[45] F. Bloch, Z. Phys. 52, 555 (1929).

[46] Maxime Ben Dahan, Ekkehard Peik, Jakob Reichel, Yvan Castin, and Maxime Christophe Salomon, Phys. Rev. Lett. 76, 4508 (1996).

[47] M. Schulze, D. Bercioux, and D. F. Urban, Phys. Rev. B 87, 024301 (2013). 